\title{
INTRODUCING A LOW-COST MINI-UAV FOR THERMAL- AND MULTISPECTRAL-IMAGING
}

\author{
J. Bendig ${ }^{\mathrm{a}, *}$, A. Bolten ${ }^{\mathrm{a}}$, G. Bareth ${ }^{\mathrm{a}, \mathrm{b}}$ \\ ${ }^{a}$ Institute of Geography (GIS \& Remote Sensing Group), University of Cologne, 50923 Cologne, Germany \\ ${ }^{\mathrm{b}}$ ICASD - International Center for Agro-Informatics and Sustainable Development (www.icasd.org) \\ (juliane.bendig, andreas.bolten, g.bareth)@uni-koeln.de
}

KEY WORDS: Agriculture, Sensor, Multisensor, Multispectral, Thermal, Imagery, Small

\begin{abstract}
:
The trend to minimize electronic devices also accounts for Unmanned Airborne Vehicles (UAVs) as well as for sensor technologies and imaging devices. Consequently, it is not surprising that UAVs are already part of our daily life and the current pace of development will increase civil applications. A well known and already wide spread example is the so called flying video game based on Parrot's AR.Drone which is remotely controlled by an iPod, iPhone, or iPad (http://ardrone.parrot.com). The latter can be considered as a low-weight and low-cost Mini-UAV. In this contribution a Mini-UAV is considered to weigh less than $5 \mathrm{~kg}$ and is being able to carry $0.2 \mathrm{~kg}$ to $1.5 \mathrm{~kg}$ of sensor payload. While up to now Mini-UAVs like Parrot's AR.Drone are mainly equipped with RGB cameras for videotaping or imaging, the development of such carriage systems clearly also goes to multi-sensor platforms like the ones introduced for larger UAVs ( 5 to $20 \mathrm{~kg}$ ) by Jaakkolla et al. (2010) for forestry applications or by Berni et al. (2009) for agricultural applications. The problem when designing a Mini-UAV for multi-sensor imaging is the limitation of payload of up to $1.5 \mathrm{~kg}$ and a total weight of the whole system below $5 \mathrm{~kg}$. Consequently, the Mini-UAV without sensors but including navigation system and GPS sensors must weigh less than $3.5 \mathrm{~kg}$. A Mini-UAV system with these characteristics is HiSystems' MK-Okto (www.mikrokopter.de). Total weight including battery without sensors is less than $2.5 \mathrm{~kg}$. Payload of a MK-Okto is approx. $1 \mathrm{~kg}$ and maximum speed is around $30 \mathrm{~km} / \mathrm{h}$. The MK-Okto can be operated up to a wind speed of less than $19 \mathrm{~km} / \mathrm{h}$ which corresponds to Beaufort scale number 3 for wind speed. In our study, the MK-Okto is equipped with a handheld low-weight NEC F30IS thermal imaging system. The F30IS which was developed for veterinary applications, covers 8 to $13 \mu \mathrm{m}$, weighs only $300 \mathrm{~g}$, and is capturing the temperature range between $-20^{\circ} \mathrm{C}$ and $100{ }^{\circ} \mathrm{C}$. Flying at a height of $100 \mathrm{~m}$, the camera's image covers an area of approx. 50 by $40 \mathrm{~m}$. The sensor's resolution is $160 \times 120$ pixel and the field of view is $28^{\circ}(\mathrm{H}) \times 21^{\circ}(\mathrm{V})$. According to the producer, absolute accuracy for temperature is $\pm 1{ }^{\circ} \mathrm{C}$ and the thermal sensitivity is $>0.1 \mathrm{~K}$. Additionally, the MK-Okto is equipped with Tetracam's Mini MCA. The Mini MCA in our study is a four band multispectral imaging system. Total weight is $700 \mathrm{~g}$ and spectral characteristics can be modified by filters between 400 and $1000 \mathrm{~nm}$. In this study, three bands with a width of $10 \mathrm{~nm}$ (green: $550 \mathrm{~nm}$, red: $671 \mathrm{~nm}, \mathrm{NIR}^{1}: 800 \mathrm{~nm}$ ) and one band of $20 \mathrm{~nm}$ width $\left(\mathrm{NIR}^{2}: 950 \mathrm{~nm}\right.$ ) have been used. Even so the MK-Okto is able to carry both sensors at the same time, the imaging systems were used separately for this contribution. First results of a combined thermaland multispectral MK-Okto campaign in 2011 are presented and evaluated for a sugarbeet field experiment examining pathogens and drought stress.
\end{abstract}

\footnotetext{
* Corresponding author.
} 


\section{INTRODUCTION}

\subsection{Motivation and Aims}

In this study, we use a point symmetrical flying UAV-system offering the advantage and balance of a stable, lightweight, lowcost, and self-manageable device in contrast to large fixed wing or helicopter-based systems. Because of technical advances in the field of drive and control technology as well as the ratio between battery capacity and battery weight the development of UAV-systems is very fast and significant. Nonami et al. (2010) as well as Ollero and Maza (2007) give reviews and summaries on available systems and definitions.

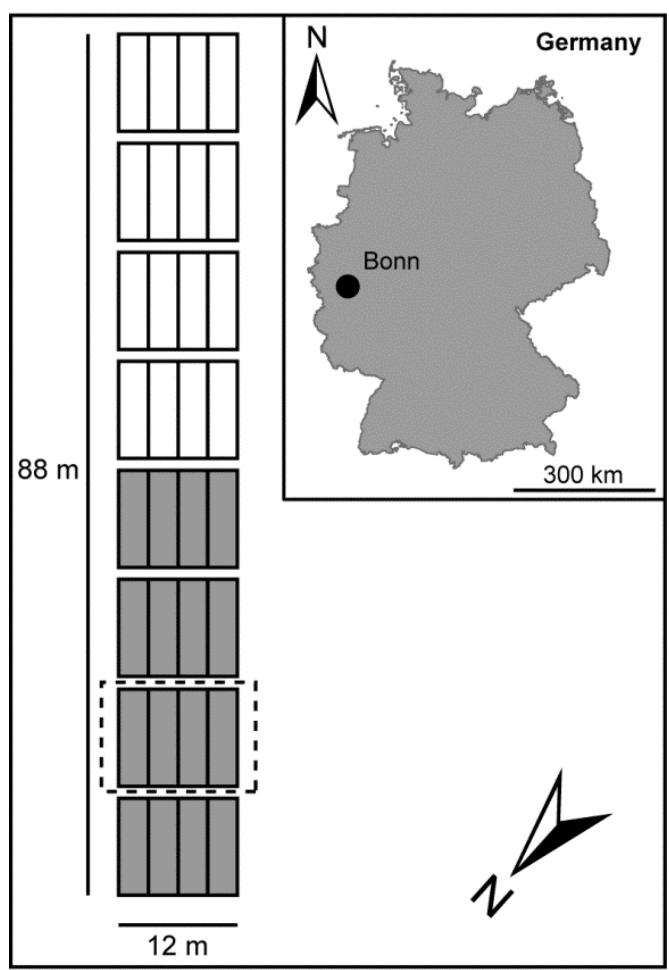

Figure 1. Orientation map and sketch of study area; white: treated plots, grey: untreated plots, dashed line: plot batch 2 (see Figure 5)

\subsection{Study Area and Dataset}

The study area is located in the city of Bonn in the west of Germany (Figure 1). In spring 2011 the Institute for Agricultural Plants and Resource Protection (INRES)Horticultural Science established an experiment field with four different types of sugar beet (AT01, AK02, AR03 and AI04) with different resistances to plant diseases. The aim of the experiment is to determine the response to natural pathogens of the different sugar beet types using non-destructive measurement techniques. First symptoms appeared 170 days after sowing. A protective and curative fungicide against three common plant diseases was applied to the control sample plants. All other horticultural activities were left unchanged. Four replications of every sugar beet type of the treated and untreated variant were planted in a randomised order with a plot size of approximately $30 \mathrm{~m}^{2}$. Ground based multispectral, chlorophyll and fluorescence measurements were carried out repeatedly during the growing season starting on day 198 after sowing. On October 13,2011, multispectral and thermal data of the four replicates of the untreated plots were collected by carrying out flights using the UAV-system.

\section{METHODS}

\subsection{Platform}

The used Oktokopter, MK-Okto by HiSystems GmbH, is a symmetrical frameset composed of aluminium and glass fibre reinforced plastics (Figure 2). The eight engines are equipped with high performance propellers. The electronics include an ARM-processor equipped mainboard, a navigation mainboard (NaviCtrl) with a compass module and a GPS module (Mikrokopter, 2012). The main Printed Circuit Board (PCB) includes high-quality gyroscopes and a pressure sensor. The use of the NaviCtrl with the pressure sensor and the GPS information enables the setting of pre-defined waypoints and altitudes to carry out autonomous flights. The electric power supply is obtained by a lithium polymer battery with up to $6600 \mathrm{mAh}$ capacity. The flight duration is around 15 minutes, depending on pay load. The flight control is realised by a standard $2.4 \mathrm{GHz}$ transmitter remote control. Additional transmitter channels are used for steering mechanisms and other functions e.g. camera triggering.

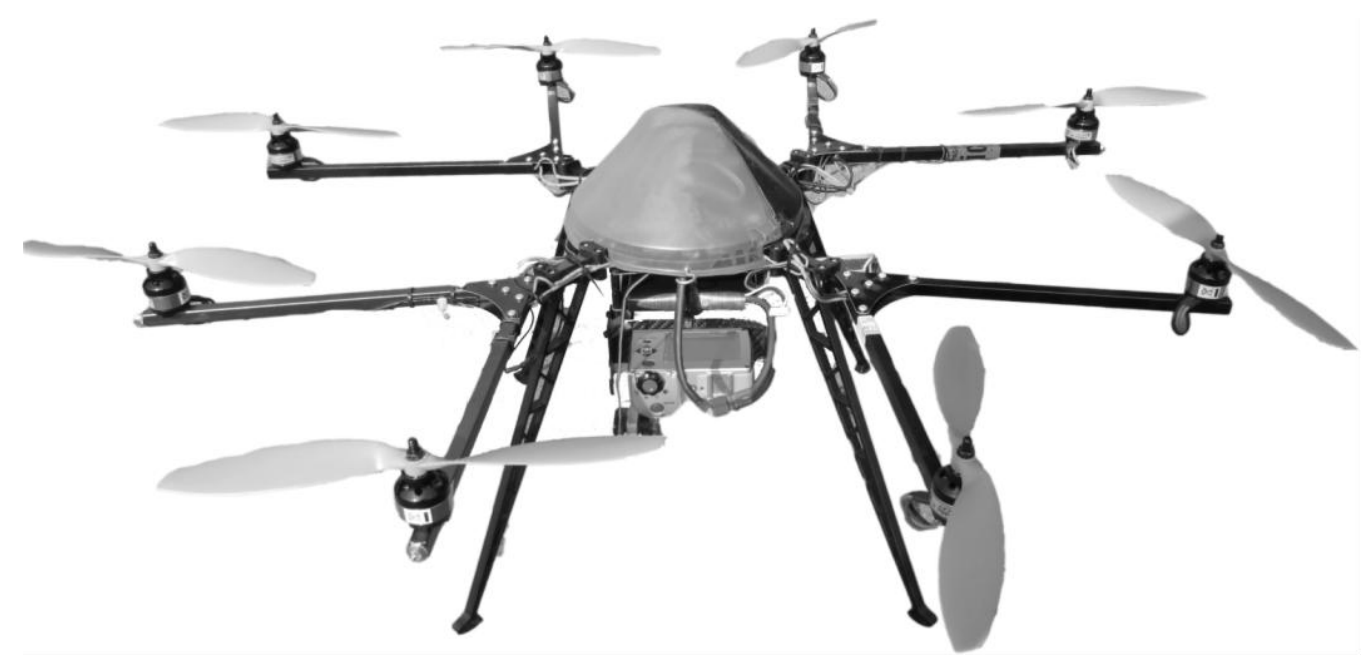

Figure 2. Mini-UAV MK-Okto by HiSystems GmbH (HiSystems GmbH, 2012) equipped with NEC F30 IS thermal imaging system 


\subsection{Sensors}

The Tetracam Mini MCA is a lightweight compact Multiple Camera Array (MCA), which consists of up to six sensors of 10 BIT data and 1.3 megapixels resolution (Figure 3, Tetracam Inc., 2012). The sensors are equipped with $25 \mathrm{~mm}$ standard spectrometer filters. The version used for this study includes four sensors with spectral values and spectral width of:

1: $550 \mathrm{~nm}(10 \mathrm{~nm})$ - Green

2: $671 \mathrm{~mm}(10 \mathrm{~nm})-$ Red

3: $800 \mathrm{~mm}(10 \mathrm{~nm})$ - Near Infrared 1

4: $950 \mathrm{~mm}(20 \mathrm{~nm})$ - Near Infrared 2

Every other filter from $450 \mathrm{~nm}$ to $1050 \mathrm{~nm}$ is possible.

The weight of the system including batteries is around $720 \mathrm{~g}$. The Field of View image size of the Mini MCA is $33 \mathrm{~m}$ by $27 \mathrm{~m}$ at a distance of $100 \mathrm{~m}$. The recording of the images is time triggered.

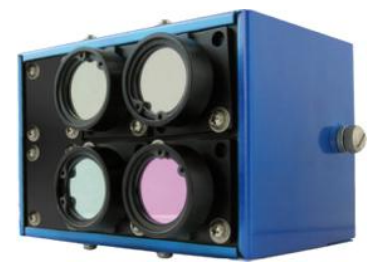

Figure 3. Tetracam Mini MCA (Tetracam Inc., 2012) multispectral camera equipped with four sensors

The NEC F30 IS is a thermal imaging system with a weight of $300 \mathrm{~g}$ and a sensor resolution of 160 by 120 pixels (Figure 4, NEC Avio Infrared Technologies Co., Ltd., 2012). The Field of View image size of the camera is $50 \mathrm{~m}$ by $37,5 \mathrm{~m}$ at a distance of $100 \mathrm{~m}$. The absolute accuracy is $\pm 1 \mathrm{~K}$ and the thermal sensitivity is around $0.1 \mathrm{~K}$. Due to the manual triggering of the camera we use an individually adapted camera holder with a mechanical trigger, operated by the remote control of the UAVsystem.

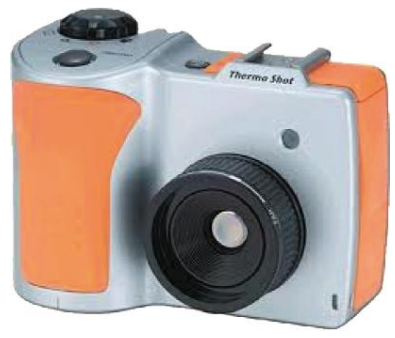

Figure 4. NEC F30 IS (NEC Avio Infrared Technologies Co., Ltd., 2012) thermal camera

\subsection{Measurement}

Prior to carrying out the flight, all corners and mid points of the plots were measured using a differential GPS. The coordinates of the mid points were transferred into a waypoint list determining the flight route of the Oktokopter.

Aluminium foil of an area of approximately half a square meter was placed on every corner to use it as a tie point in both the thermal and multispectral images to facilitate georeferencing of the data. On the first flight, the UAV was mounted with the NEC F30 IS thermal imaging system. For the second flight it was equipped with Tetracam's Mini MCA. During the flights several nadir pictures per plot with a size of $18 \mathrm{~m}$ by $15 \mathrm{~m}$ were recorded at an altitude of $55 \mathrm{~m}$ with a constant orientation.
For both imaging systems proprietary software is used to download and preprocess the datasets. After that, the main part of the data analysis was carried out using Esri's ArcGIS.

Thermal datasets were exported as ASCII data and imported into ArcMap. Georeferencing was done by using the coordinates of the measured tie points. The RAW multispectral data was converted to MultiTIF data which was then broken down into single TIF files per band. Every single file was then georeferenced using ArcMap. Combination of both datasets in the GIS allows for comparison of the data to detect special characteristics e.g. infield variability. Furthermore, NDVI (Meer \& de Jong, 2001) was calculated from the multispectral data using ArcGIS.

\section{RESULTS AND DISCUSSION}

\subsection{General}

The altitude for both flights was $55 \mathrm{~m}$ above ground in clear sky conditions. Thermal images with 120 by 160 pixels and multispectral images with 1280 by 1024 pixels before georeferencing were recorded. Ground resolution for thermal data is $17 \mathrm{~cm}$ and $1.4 \mathrm{~cm}$ for multispectral data. The size of 60 by $60 \mathrm{~cm}$ chosen for the tie points is appropriate.

The GPS data has an overall accuracy of $1 \mathrm{~cm}$ which is satisfactory to ensure exact positioning of the UAV-system. Considering the resolution of the produced pictures, the accuracy of the tie points is also appropriate.

Georeferencing of every single file worked well for the thermal data and the green and red channel of the multispectral data. For the NIR channels the reflection of the tie points produced a lower contrast making the georeferencing more challenging. The use of an additional material with different reflection behavior will be considered for future measurements.

\subsection{Multispectral Data and Thermal Data}

In the following section data of plot batch 2 (see Figure 1) will be presented.

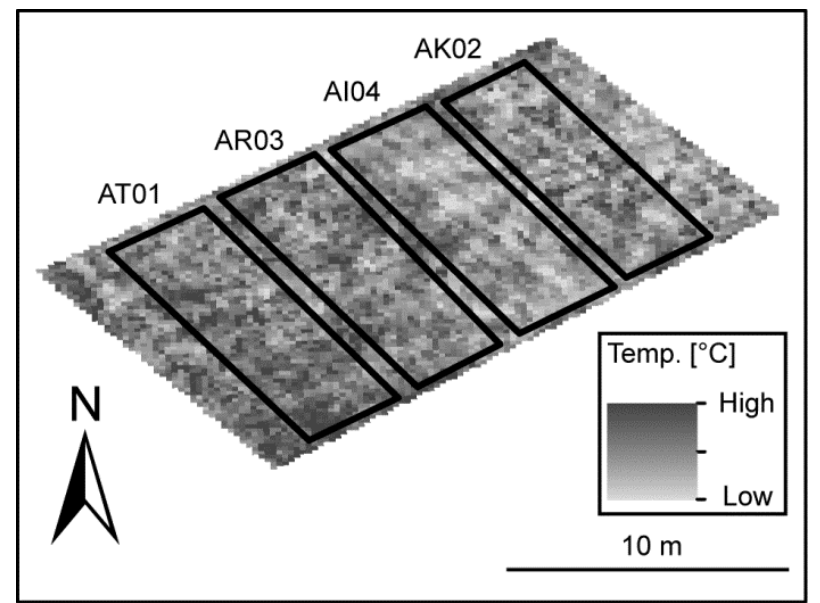

Figure 5. Measured temperature $\left[{ }^{\circ} \mathrm{C}\right]$ of different sugar beet types in plot batch 2 (see Figure 1)

Infield variability can be detected both in the multispectral data and the thermal data (Figure 5). The comparison of NDVI and temperature between the different sugar beet types reveals more detailed information (Table 1, Figure 6 and 7):

The histogram of the calculated NDVI shows a similar curve progression with four peaks $(0.42-0.46 ; 0.48-0.54 ; 0.57-0.64$; 
0.69-0.78) with detectable differences between the sugar beet types. AR03 and AI04 have a similar NDVI distribution while AT01 has lower frequencies at peaks three and four. AK02 has lower frequencies at peaks one to three compared to the others. Minimum NDVI varies from 0.28 to 0.37 and mean NDVI from 0.66 to 0.67 ; while Maximum NDVI is 0.78 (Table 1).

The analysis of the histogram for the thermal data allows for detection of similar characteristics for AT01 and AR03 (Figure 7). The peak temperatures of AI04 are located at lower temperatures $\left(12.0-12.8^{\circ} \mathrm{C}\right)$ compared to the other sugar beet types $\left(12.3-13.2{ }^{\circ} \mathrm{C}\right)$. The curve progression of AK02 is more balanced over the whole temperature spectrum $\left(11.2-15.10{ }^{\circ} \mathrm{C}\right)$ compared to the other samples. Mean temperatures also indicate differences between the samples $\left(12.52-13.03{ }^{\circ} \mathrm{C}\right.$; Table 1$)$.

\begin{tabular}{|c|c|c|c|c|}
\hline & AT01 & AK02 & AR03 & AI04 \\
\hline \multicolumn{5}{|l|}{ Multispectral: NDVI } \\
\hline Min & 0.28 & 0.37 & 0.36 & 0.30 \\
\hline $\operatorname{Max}$ & 0.78 & 0.78 & 0.78 & 0.78 \\
\hline Mean & 0.66 & 0.69 & 0.67 & 0.67 \\
\hline Standard deviation & 0.09 & 0.07 & 0.08 & 0.08 \\
\hline \multicolumn{5}{|c|}{ Thermal: Temperature $\left[{ }^{\circ} \mathrm{C}\right]$} \\
\hline Min & 11.40 & 11.20 & 11.40 & 11.30 \\
\hline $\operatorname{Max}$ & 15.10 & 14.70 & 14.80 & 14.30 \\
\hline Mean & 13.03 & 12.67 & 12.85 & 12.52 \\
\hline Standard deviation & 0.59 & 0.60 & 0.56 & 0.52 \\
\hline
\end{tabular}

Table 1. Statistics of different sugar beet types in plot batch 2 (see Figure 1)

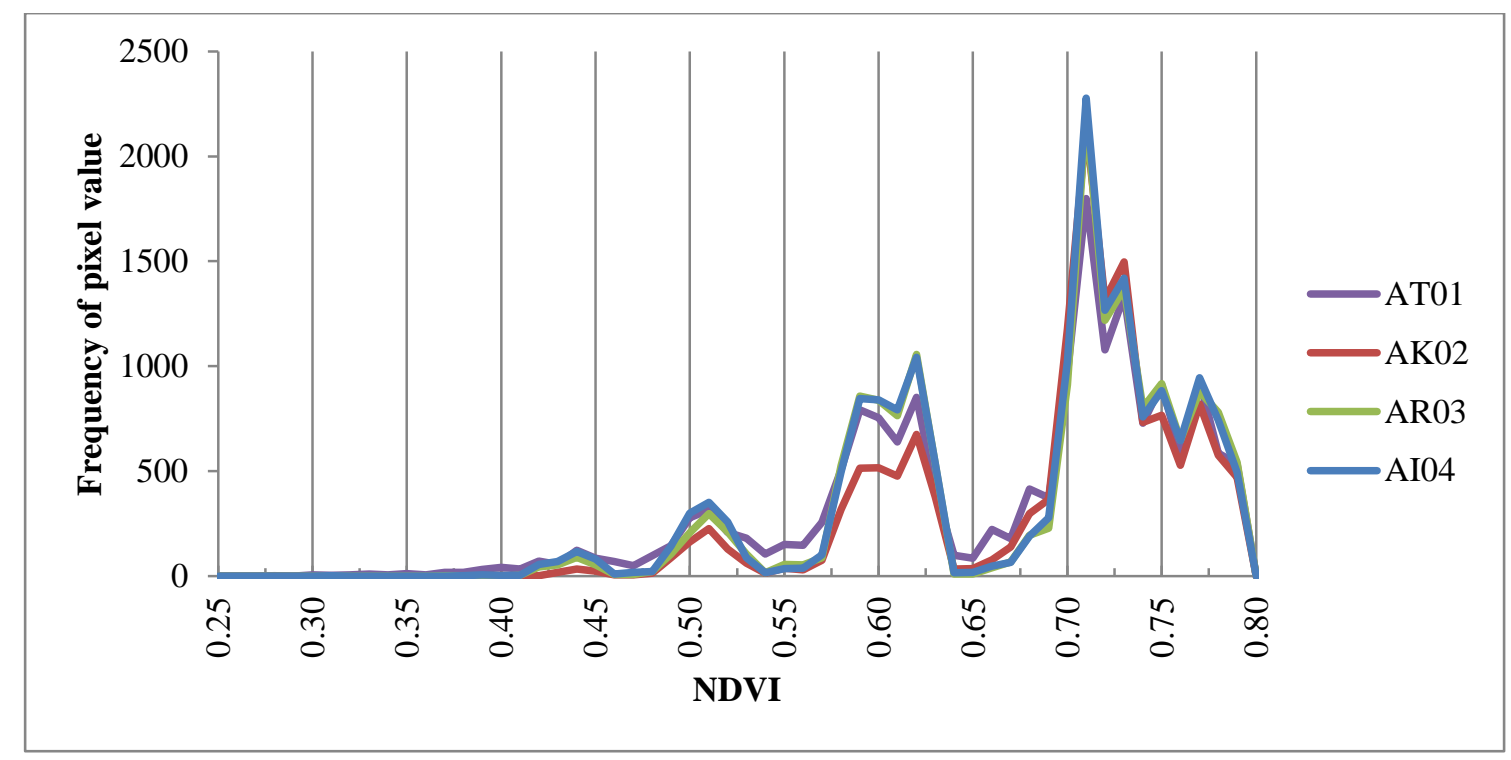

Figure 6. Histogram of measured NDVI values of different sugar beet types in plot batch 2 (see Figure 1)

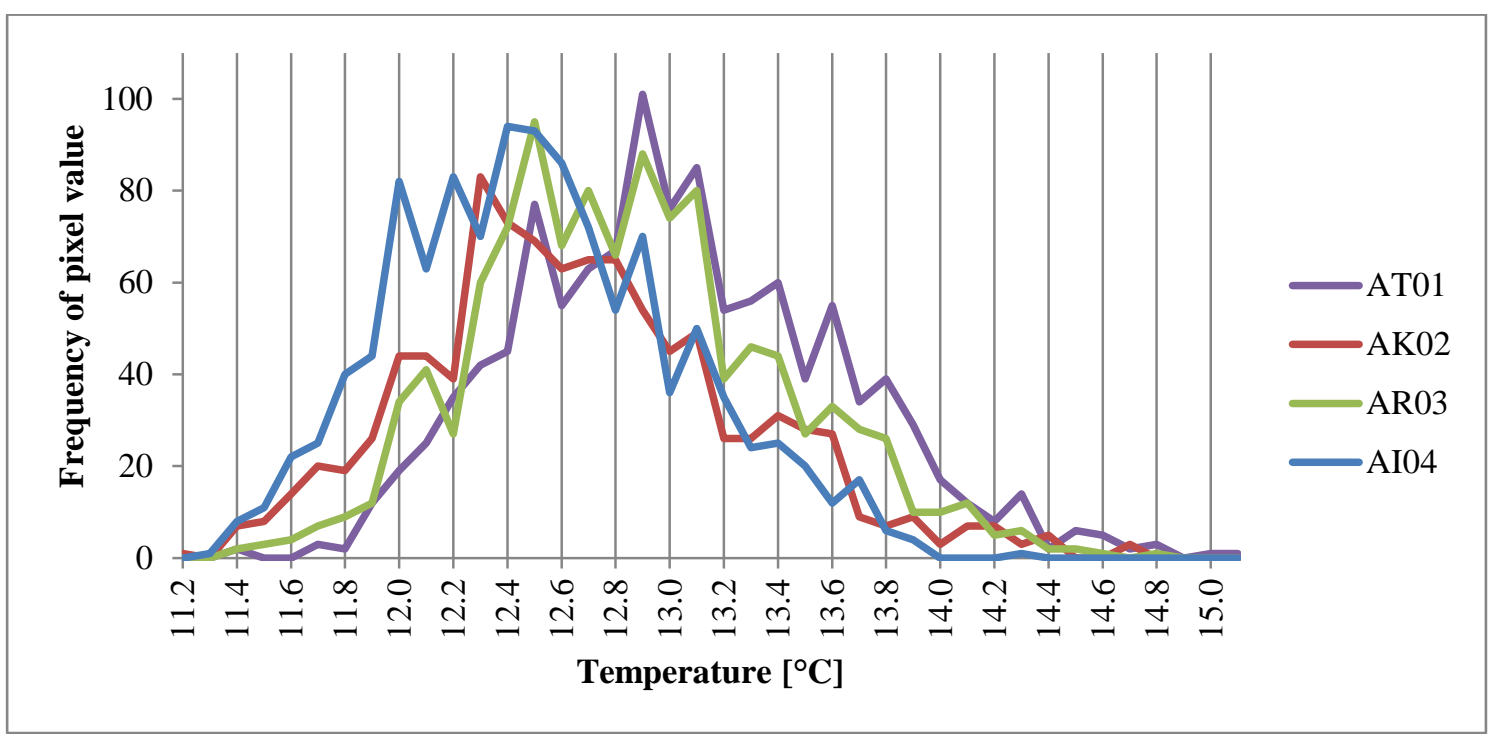

Figure 7. Histogram of measured temperature $\left[{ }^{\circ} \mathrm{C}\right]$ of different sugar beet types in plot batch 2 (see Figure 1) 


\subsection{Suitability of the Platform}

The results presented above show that the MK-Okto, a low-cost Mini-UAV platform, is suitable for the acquisition of thermal and multispectral images. The georeferencing of tie points in the data and autonomous flights under standardised conditions e.g. defined waypoints and altitudes ensure the comparability of the data.

For small scale applications, like the experiment field in this contribution, the flight time of about 15 minutes is sufficient.

Since the platform is built and maintained directly by the user it can be utilised for various sensors, only limited by the pay load of $1 \mathrm{~kg}$.

\section{OUTLOOK}

Future tasks will be to validate the current results with additional measurements. Thus, a multitemporal dataset can be created in order to enable further analysis. For this purpose calibration panels for the multispectral measurements were built and will be used in future flight campaigns (see Laliberte (2011) or Hunt et al. (2011)).

In addition, the Lumix GF3 RGB camera, another sensor will be used on the platform, enabling the generation of stereo images. See Aber et al. (2010), Eisenbeiss et al. (2005) and Eisenbeiss and Sauerbier (2011) for examples on DEM generation from stereo images collected by UAV.

Furthermore, a combination of the data collected with different ground measurements such as hyper-spectral data or chlorophyll data is possible to obtain. This also allows for additional evaluation of the data collected with the Mini-UAV.

The aim of research is to produce a multisensoral, multitemporal dataset, focusing on exploring the suitability of the Oktokopter as a multi-sensoral platform.

\section{ACKNOWLEDGEMENTS}

The authors would like to thank the Institute for Agricultural Plants and Resource Protection (INRES)-Horticultural Science of University of Bonn (Prof. Dr. Georg Noga, Dr. Mauricio Hunsche and Georg Leufen), for providing the experiment field and general cooperation.

\section{REFERENCES}

Aber, J. S., Marzloff, I., Ries, J. B., 2010: Small-Format Aerial Photography. Principles, Techniques and Gesoscience Applications. Elsevier, Amsterdam.

Berni, J. A. J., Zarco-Tejada, P. J., Suárez, L., Fereres, E., 2009. Thermal and Narrowband Multispectral Remote Sensing for Vegetation Monitoring From an Unmanned Aerial Vehicle. IEEE Transactions on Geoscience and Remote Sensing, 47(3), pp. 722-738.

Eisenbeiss, H., Sauerbier, M., 2011. Investigation of uav systems and flight modes for photogrammetric applications. The Photogrammetric Record, 26(136), pp. 400-421.

Eisenbeiss, H., Sauerbier, M., Zhang, L., Grün A., 2005. Mit dem Modellhelikopter über Pinchango Alto. Géomatique Suisse, 9, pp. 510-515.
HiSystems GmbH, 2012. HiSystems GmbH. http://www.hisystems.de (05 Mar. 2012).

Hunt, E. R., Hively, W. D., McCarty, G. W., Daughtry, C. S. T., Forrestal, P. J., Kratochvil, R. J., Carr, J. L., Allen, N. F., FoxRabinovitz, J. R., Miller, Ch. D., 2011. NIR-Green-Blue HighResolution Digital Images for Assessment of Winter Cover Crop Biomass. GIScience \& Remote Sensing, 48(1), pp. 86-98.

Jaakkola, A., Hyyppä, J., Kukko, A., Yu, X., Kaartinen, H., Lehtomäki, M., Lin, Y., 2010. A low-cost multi-sensoral mobile mapping system and its feasibility for tree measurements. ISPRS Journal of Photogrammetry and Remote Sensing, 65, pp. 514-522.

Laliberte, A. S., Goforth, M. A., Steele, C. M., Rango, A., 2011. Multispectral Remote Sensing from Unmanned Aircraft: Image Processing Workflows and Applications for Rangeland Environments. Remote Sensing, 3, pp. 2529-2551.

Meer, F. D., de Jong, S. M. (Eds.), 2001. Imaging Spectrometry. Basic Principles and Prospective Applications. Kluwer Academic Publishers, Dordrecht.

Mikrokopter, 2012. NaviCtrl V2.0.

http://www.mikrokopter.de/ucwiki/NaviCtrl_2.0

(28 Feb. 2012).

NEC Avio Infrared Technologies Co., Ltd., 2012. Thermo Shot F30 Series/F20: Specifications.

http://www.nec-avio.co.jp/en/products/ir-

thermo/lineup/f30/spec.html (28 Feb. 2012).

Nonami, K., Kendoul, F., Suzuki, S., Wang, W., Nakazawa, D., 2010. Autonomous flying robots. Springer, Tokyo.

Ollero, A., Maza, I., 2007: Multiple heterogeneous unmanned aerial vehicles. Springer, Berlin.

Tetracam Inc., 2012. MCA Series - High Quality Multi-Spectral Imaging. Mini MCA.

http://www.tetracam.com/mca_mini.html (28 Feb. 2012). 